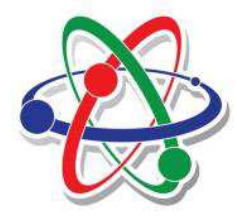

Open Medscience

Peer-Reviewed Open Access

JOURNAL OF DIAGNOSTIC IMAGING IN THERAPY

Journal homepage: www.openmedscience.com

Editorial Review

\title{
Editorial Review 2015 - Nuclear Medicine, Diagnostic Imaging and Therapy
}

\section{Andrea Ciarmiello ${ }^{1}$ and Luigi Mansi ${ }^{2}$}

${ }^{1}$ Nuclear Medicine Department, S. Andrea Hospital, La Spezia, Italy

${ }^{2}$ Nuclear Medicine Unit, Department of Clinical and Experimental Internistic 'F.Magrassi, A.Lanzara', Seconda Università di Napoli, Napoli, Italy

Author to whom correspondence should be addressed:

Andrea Ciarmiello, M.D.

Editor-in-Chief

Journal of Diagnostic Imaging in Therapy

andrea.ciarmiello@as15.liguria.it

Keywords: nano-technology; radiotherapy; PET imaging; hypoxia imaging; dosimetry; cyberknife; tomotherapy; nano-aptamers; Alzheimer's plaque imaging

Journal of Diagnostic Imaging in Therapy (JDIT) is published online by Open Medscience, based in Northern Ireland, UK. The aim of this journal is to address the requirements of researchers specialising in nuclear medicine, diagnostic imaging and therapy by providing open access to peerreviewed articles. These high quality published articles are available in both HTML and PDF formats. All published articles are assigned an unique CrossRef DOI number and the HTML version is given a CrossMark accreditation. 
The published articles highlight the application of diagnostic imaging with radionuclides, X-rays, magnetic resonance (MR), ultrasound (US) etc. The scope of these imaging modalities include: positron emission tomography (PET), single photon emission computed tomography (SPECT), hybrid imaging systems, radioguided surgery (RGS) and positron emission mammography (PEM). Also included are the application of short and long-lived radioisotopes in research alongside the development of imaging agents and related targeted therapies. In addition, JDIT's scope will include magnetic resonance imaging (MRI), computed tomography (CT), ultrasound (US) imaging and planar $\mathrm{X}$-ray (digital, analogue and portable) systems [1].

Articles published in 2015 have covered the topics on the application of nano-particles towards imaging and therapy, quantification of PET/CT images, the role of $\left[{ }^{18} \mathrm{~F}\right] \mathrm{FDG}$ positron emission tomography (PET) imaging for glucose transporter GLUT1, bifunctional metal - nitroimidazole complexes for hypoxia theranosis in cancer, 3-D image-based dosimetry using ${ }^{90} \mathrm{Y}$ microsphere therapy, reirradiation of spinal metastases using micro multileaf collimator, nano-aptamer for breast cancer imaging and gallium-68 radiotracers for Alzheimer's plaque imaging.

These published articles have all been peer-reviewed by the journals' editorial board and/or external reviewers. Here, we would personally like to take this opportunity to thank everyone on the journals' editorial board who has volunteered their time to review these articles.

In this editorial review, we have summarized all the abstracts from the 2015 issue.

\section{Nano-technology}

The first article of 2015 to be published online in the Journal of Diagnostic Imaging in Therapy was on the subject 'Polymeric nano-hydroxyapatite coated with polylactic acid (PLA): considering new possibilities for radiopharmacy', by Santos-Oliveira et al. [2].The authors described a technique which utilises a polymer based on lactic acid to encapsulate nano-hydroxyapatite. These materials will provide new applications in the areas of oncology and radiopharmacy. The aim of this technology is to link certain radionuclides and other vectors such as aptamers to create therapies which contain nanoparticles. These nanomaterial platforms will enable the delivery of radiopharmaceuticals to diagnose and destroy tumours. Continued investigations are required to establish the ability of nanohydroxyapatite structures and their impact on nuclear medicine, imaging and therapeutic areas.

\section{Radiotherapy}

The next article on 'PET/CT images quantification for diagnostics and radiotherapy applications', by Ferrando et al. for the detection and staging of various malignant tumours [3]. The authors routinely applied Standard Uptake Value (SUV) in clinical oncology and generated images for target volume definition in the radiotherapy planning stages. A limitation of PET is the resolution of the system even though PET is a powerful diagnostic tool. The authors of this article present an analysis of a phantom study to clarify the accuracy of the generated data from the tomography including the validation method used in radiotherapy. This IEC phantom study was carried out using the PET/CT hybrid 
system. The spheres with different sphere/background activity ratios $\left(\mathrm{R}_{\mathrm{S} / \mathrm{B}}\right)$ and the volume was calculated using an adaptive thresholding method. The SUV obtained in each sphere gave the percentage error relative to the real values. Subsequently, the hot contrast recovery coefficients (HCRC) obtained a linear relationship between the threshold volumes up to $10 \mathrm{~mL}$. The threshold volumes between $10 \mathrm{~mL}$ and $5.5 \mathrm{~mL}$ decreased reaching a minimum volume at $1.1 \mathrm{~mL}$. Volumes less than $1.1 \mathrm{~mL}$, increase exponentially and no dependence on the acquisition time was observed. Subsequently, the thresholds depend on sphere volumes and $\mathrm{R}_{\mathrm{S} / \mathrm{B}}$, including smoothing filter. The SUV values were quantified to certain volumes. In summary, for objects with volumes of less than $2.5 \mathrm{~mL}$ the SUV values were significantly effected with an error up to $80 \%$. From the clinical point-of-view, a false negative result can be derived from very small lesions due to low measured SUVmax value. In addition, the limited PET resolution influences lesion segmentation and therefore an adaptive thresholding method is a useful tool for tumour boundary definition. The potential limitation is for unreliable results with volumes less than $2.5 \mathrm{~mL}$.

\section{PET Imaging}

In this article entitled, 'Roles of facilitative glucose transporter GLUT1 in $\left[{ }^{18} \mathrm{~F}\right] \mathrm{FDG}$ positron emission tomography (PET) imaging of human diseases', by Simon Patching [4]. PET imaging of human disease states can benefit from the glucose transport protein GLUT1, due to its widespread expression in cellular systems. In addition, the catalyst role to facilitate the diffusion of glucose across red blood cell membranes. This extends to the blood-brain barrier including membranes of some organelles. PET imaging techniques can measure the uptake of $\left[{ }^{18} \mathrm{~F}\right] \mathrm{FDG}$ into cells and tissues and therefore acts as a marker for glucose transport, including glycolytic activity. Various disease states can alter the glycolytic activity in localised regions of tissues or organs, which can be visualised using $\left[{ }^{18} \mathrm{~F}\right] \mathrm{FDG}$ PET. The activity levels associated with GLUT1 contribute to the pattern and intensity of $\left[{ }^{18} \mathrm{~F}\right] \mathrm{FDG}$ PET imaging used in diagnosing and monitoring a range of human diseases. The proliferation of cancer cells display an overexpression of GLUT1 and an increased rate of glycolysis due to increased nutrient demands. Therefore, tumours have enhanced $\left[{ }^{18} \mathrm{~F}\right] \mathrm{FDG}$ uptake compared to normal cells, so $\left[{ }^{18} \mathrm{~F}\right] \mathrm{FDG}$ PET is routinely used in diagnosing and monitoring of various cancer types. PET imaging of the brain is useful in the identification of hypometabolism and/or hypermetabolism associated with neurological disorders such as Alzheimer's disease, Parkinson's disease, epilepsy, schizophrenia, multiple sclerosis and cerebral ischemia. These PET imaging techniques can be extended to other conditions including cardiovascular diseases, inflammation, sarcoidosis, atherosclerosis, and infections.

\section{Hypoxia Imaging}

The review article on, 'Bifunctional Metal - Nitroimidazole Complexes for Hypoxia Theranosis in Cancer', by Ricardo et al. discusses the design, radiochemistry and hypoxia-selective properties of organometallic complexes including nitroimidazoles, towards bioactive targets [5]. Numerous drugs are based on the substrate 2-nitroimidazole and its ability to be effective radiosensitizers of hypoxic cells. 
These nitroimidazole derivatives can undergo initial oxygen-reversible, enzymatic one-electron reductions that can lead to the formation of molecular adducts that inhibit vital molecular processes. The accumulation of radiolabelled adducts within hypoxic cells allow for imaging and calculations regarding the radiopharmaceutical concentration. The imaging and therapeutic potential of hypoxiatargeted organometalic nitroimidazole derivatives depend on the type of radiometal used. The selected ligands consist of a broad range of mono- or poly-dentate, linear or cyclic chelators. These ligands are adapted to create hypoxia-selective nitroimidazoles or nitrotriazoles using various modified linker technologies. The resultant metal-nitroimidazole complexes possess reducible centres and can produce redox properties. These properties have the ability to result in interactions inside the target (hypoxic) and normoxic tissues. In conclusion, these complexes which contain reducible metal cores, including reducible targeting vectors (nitroimidazole), can allow for greater selectivity and sensitivity for hypoxic tissues. This is compared to either reducible metal-complexes on their own or the nitroimidazole without the reducible metal centre.

\section{Dosimetry}

The article, 'A review of 3D image-based dosimetry, technical considerations and emerging perspectives in ${ }^{90} \mathrm{Y}$ microsphere therapy', by Jim O'Doherty [6]. This therapy which involves yttrium90 radioembolization $\left({ }^{90} \mathrm{Y}-\mathrm{RE}\right)$ is used in the treatment of hepatocellular carcinoma and other disease states. Nuclear Medicine and Cath Lab diagnostic imaging have a major role in the treatment plan of patients. The effective treatment plan can only be developed by generating robust dosimetry data at the various clinical stages to derive an effective personalized medicine plan. In this review, the current

${ }^{90} \mathrm{Y}-\mathrm{RE}$ techniques are outlined and the challenges in generating the quantification and dosimetry data are discussed. The author also focuses on the current 3-D dosimetry techniques, including highresolution imaging and novel surgical procedures. In addition, the use of other radiopharmaceuticals for therapy and therapeutic planning are disscussed.

\section{Cyberknife and Tomotherapy}

In this article, 'Reirradiation of spinal metastases using an add-on double-focus micro multileaf collimator and a three partial-arc conformal avoidance technique with optimized beam weights: a planning study', by Nishiyama et al. This article discusses the role of the Cyberknife and tomotherapy in the reirradiation of spinal metastases and the importance of dose delivery [7]. The use of the Cyberknife requires longer treatment time and can produce effects on the patient regarding immobility. The other factors involve tomotherapy are that it is capable in delivering rotational beams of radiation dose which may put the surrounding organs at risk (OARs). Therefore, the application of stereotactic body radiotherapy was successfully employed for the reirradiation which allowed for high dosages to the target without damaging the function of the spinal cord and neighbouring OARs.

\section{Nano-Aptamers}

The penultimate article, 'Nano-Aptamer for Breast Cancer Imaging: Initial Considerations', by Santos do Carmo et al. [8]. The design of imaging and therapy agents based on nanoparticles towards the application of aptamers will enhance the development of novel drug delivery systems. Nano- 
technology involving radiopharmaceuticals have the potential to solve several problems regarding the in vivo stability of aptamers. The authors address the development and proof-of-concept of nanoaptamers with the aim in supporting its application as a nano-radiopharmaceutical, for the treatment of breast cancer and other disease states.

\section{Alzheimer's plaque imaging}

The final article of 2015 was on 'Gallium-68 radiotracers for Alzheimer's plaque imaging', by BarriosLopez et al. [9]. The application of imaging tools towards the diagnosis of the brain disorder Alzheimer's disease (AD). This type of dementia is increasing in the aging population, and imaging tools of $\beta$-amyloid $(\mathrm{A} \beta)$ plaques are vital for clinical and neuropsychological characteristics in AD. Imaging techniques allows for better understanding in the progression of this disease and the development of new drug treatments. PET imaging can be used in the development of drugs at the different clinical stages due to its high sensitivity and capacity to produce pharmacokinetic data. The PET imaging agent Pittsburgh $\mathrm{P}\left({ }^{11} \mathrm{C}-\mathrm{PIB}\right)$ can bind onto amyloid $\mathrm{A} \beta$ plaques, and other PET imaging probes are in clinical trials. These include thioflavin $\mathrm{T},{ }^{11} \mathrm{C}-\mathrm{SB}-13,{ }^{18} \mathrm{~F}-\mathrm{GE}-067,{ }^{18} \mathrm{~F}-\mathrm{AZD} 4694,{ }^{18} \mathrm{~F}$ BAY94-9172 and ${ }^{18} \mathrm{~F}-\mathrm{AV}-45$. PET imaging can be ulitised for in vivo $\beta$-amyloid plaques detection. The aim is to develop PET tracers, based on ${ }^{68} \mathrm{Ga}$ chemistry. The characteristics of ${ }^{68} \mathrm{Ga}$ are that it decays by $89 \%$ over positron emission of $1.9 \mathrm{MeV}$ and $11 \%$ orbital electron capture and the associated half-life is $67.7 \mathrm{~min}$. Other advantages include long half-life of the ${ }^{68} \mathrm{Ge} /{ }^{68} \mathrm{Ga}$ generator system, that is 270 days. This article also focuses on the use of gallium-68 radiotracers used for human amyloid imaging.

This journal continues to capture the imagination of many scientists working in nuclear medicine, diagnostics, imaging and therapy. The journal owes its success to the high quality manuscripts that are available on the open access platform to everyone.

\section{Prof. Andrea Ciarmiello}

Editor-in-Chief

Journal of Diagnostic Imaging in Therapy

\section{Prof. Luigi Mansi}

Co-Editor-in-Chief:

Journal of Diagnostic Imaging in Therapy

\section{Articles}

[1] Ciarmiello A, Mansi L. Inaugural Editorial Review: Nuclear Medicine, Diagnostic Imaging and Therapy. Journal of Diagnostic Imaging in Therapy. 2015; 2(1): 1-8. [CrossRef]

[2] de Souza Albernaz M, Gilberto Weissmuller G, Linhares Rossi A, Malta Rossi A, Santos-Oliveira R. Polymeric nano-hydroxyapatite coated with polylactic acid (PLA): considering new possibilities for radiopharmacy. Journal of Diagnostic Imaging in Therapy. 2015; 2(1): 9-17. [CrossRef] 
[3] Ferrando O, Foppiano F, Scolaro T, Gaeta C, Ciarmiello A. PET/CT images quantification for diagnostics and radiotherapy applications. Journal of Diagnostic Imaging in Therapy. 2015; 2(1): 18-29. [CrossRef]

[4] Patching SG. Roles of facilitative glucose transporter GLUT1 in $\left[{ }^{18} \mathrm{~F}\right] \mathrm{FDG}$ positron emission tomography (PET) imaging of human diseases. Journal of Diagnostic Imaging in Therapy. 2015; 2(1): 30-102. [CrossRef]

[5] Ricardo CL, Kumar P, Wiebe LI. Bifunctional metal - nitroimidazole complexes for hypoxia theranosis in cancer. Journal of Diagnostic Imaging in Therapy. 2015; 2(1): 103-158. [CrossRef]

[6] O' Doherty J. A review of 3D image-based dosimetry, technical considerations and emerging perspectives in ${ }^{90} \mathrm{Y}$ microsphere therapy. Journal of Diagnostic Imaging in Therapy. 2015; 2(2): 1-34. [CrossRef]

[7] Nishiyama S, Yoda K, Komatsu T. Reirradiation of spinal metastases using an add-on double-focus micro multileaf collimator and a three partial-arc conformal avoidance technique with optimized beam weights: a planning study. Journal of Diagnostic Imaging in Therapy. 2015; 2(2): 35-40. [CrossRef]

[8] Santos do Carmo F, Rocha Pinto S, Maria Camões Orlando M, et al. Nano-aptamer for breast cancer imaging: initial considerations. Journal of Diagnostic Imaging in Therapy. 2015; 2(2): 41-49. [CrossRef]

[9] Barrios-Lopez B, Airaksinen A, Bergström K. Gallium-68 radiotracers for Alzheimer's plaque imaging. Journal of Diagnostic Imaging in Therapy. 2015; 2(2): 50-65. [CrossRef]

Citation: Ciarmiello A, Mansi L. Editorial Review 2015 - Nuclear Medicine, Diagnostic Imaging and Therapy. Journal of Diagnostic Imaging in Therapy. 2016; 3(1): 1-6.

DOI: http://dx.doi.org/10.17229/jdit.2016-0116-020

Copyright: (C) 2016 Ciarmiello A, Mansi L. This is an open-access article distributed under the terms of the Creative Commons Attribution License, which permits unrestricted use, distribution, and reproduction in any medium, provided the original author and source are cited.

Received: 7 January 2016 | Revised: 13 January 2016 | Accepted: 16 January 2016

Published Online 16 January 2016 http://www.openmedscience.com 